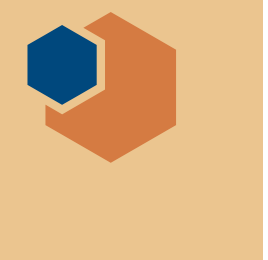

\title{
US Air Force supports high-flying science research in Asia-and around the world
}

www.SAM.gov

$\mathrm{O}$ pportunities for research funding from the US military do not stop at the nation's borders. In keeping with the globalization of science, military programs like those sponsored by the US Air Force Office of Scientific Research (AFOSR), cast a wide net in selecting research projects - including materials science research - for funding and other support.

AFOSR's international programs include its Asian Office of Aerospace Research and Development (AOARD), headquartered in Tokyo. Director Kenneth Caster says the office covers the entire Asia-Pacific region where all researchers at eligible academic or research institutions and who have an active registration with the US government may participate. Details on eligibility, he says, can be found at www.SAM.gov.

AOARD is one of AFOSR's three international offices, the others being the European Office of Aerospace Research and Development and the Southern Office of Aerospace Research and Development. There is no grant cycle, AFOSR says - proposals are accepted year-round through open Broad Agency Announcements available at www.grants.gov.

Within AOARD, materials research is concentrated on two main topics. The first is structural materials as applied to load-bearing structures, particularly for

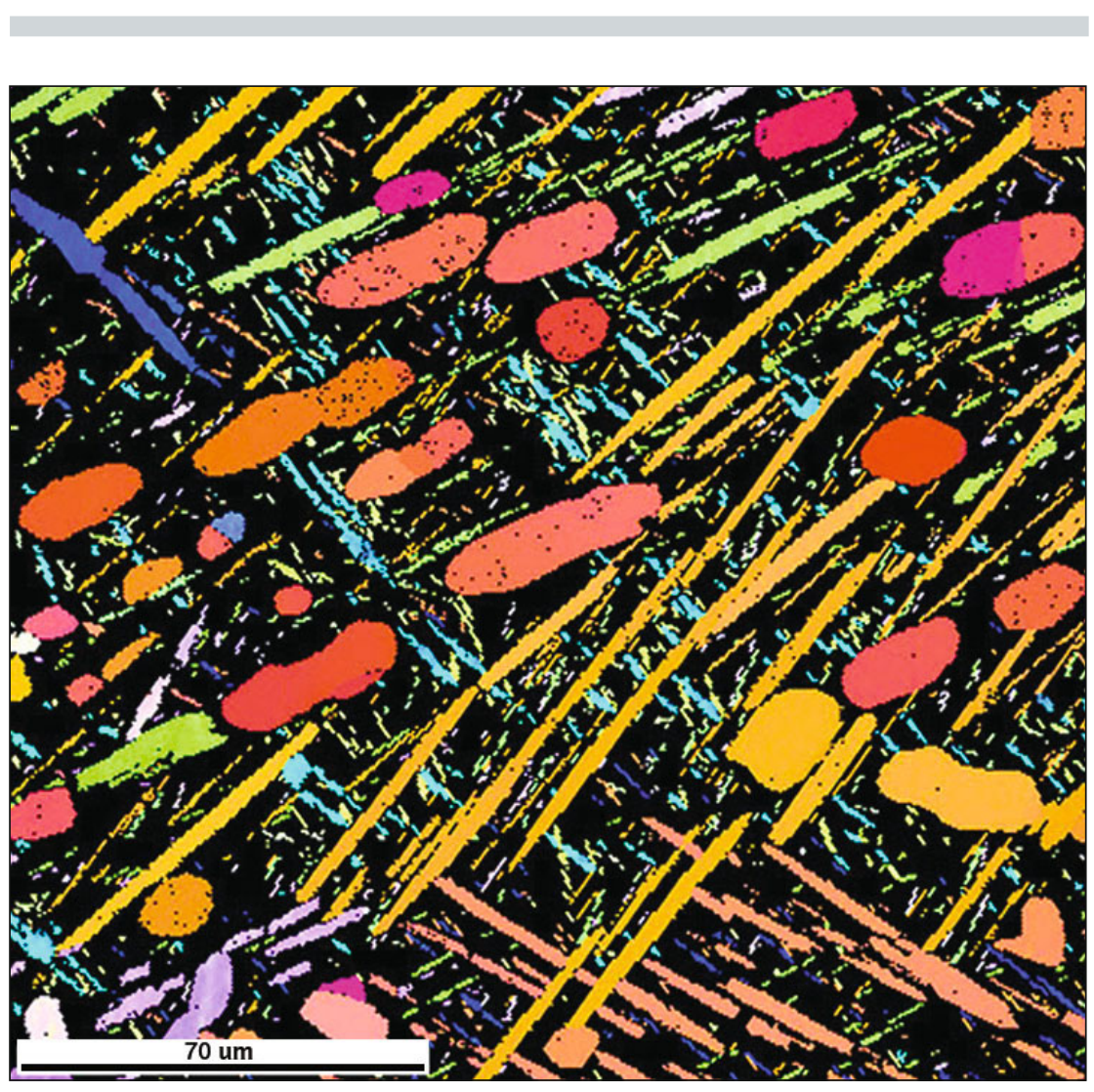

Thermomechanically processed Ti alloys from the research group of Dipankar Banerjee of the Indian Institute of Science, Bangalore. air and space applications. Particular focus areas, AOARD says, include metals, ceramics, composites, and "compliant" or soft materials, as well as novel processing techniques.

The other topic is materials discovery and manufacturing science, including exploration of new materials systems and the use of autonomous experimentation for materials discovery and manufacturing optimization. Materials systems of particular interest currently are high-entropy alloys, magnetic materials, programmable materials, flexible electronics, materials for extreme environments, multifunctional materials, and nanoscale materials and structures.

There are several reasons to work with international researchers, including researchers in the Asia-Pacific region, Caster says. First, basic research is international with about $80 \%$ occurring outside of the United States. Also, AFOSR wants to leverage large, national, and regional governmental investments, in particular research areas such as nanosciences and nanomaterials. That includes access to specialized and unique instruments and test capabilities, he says, for example Australia's cyclotron - the only one of its kind in the world. And finally, he says, there is the intrinsic value of collaborating with researchers and institutes with different perspectives but similar goals.

Specific to the US Air Force, Caster says, "the goal is to develop materials in order to advance air, space, and cyber [technologies] and to prevent technical surprise."

The professional linkages forged by AFOSR program officers and between researchers and collaborators themselves fuel interest in - and grant applications for-the monies available, according to researchers who have received grant funding from AOARD. 
Francesca Iacopi, a professor of electrical engineering at the University of Technology Sydney, says she learned about AOARD funding through a visit by Caster to her university. Caster, she says, gave a presentation about AOARD and AFOSR, including the possibility for funding research groups outside of the United States.

Iacopi says she had already been working with a colleague from the US Air Force Research Laboratory, John Boeckl, "who is nowadays one of my closest collaborators. We started working together organically, as we were both interested in graphene and silicon carbide, and had very complementary expertise which created a great synergy."

"I have two projects with AOARD," says grant recipient Dipankar Banerjee, honorary professor of organic chemistry at the Indian Institute of Science, Bangalore. He says his group aims to construct both "a quantitative and predictive understanding of the phenomena underlying the materials development and application effort with an emphasis on engineering materials, and especially titanium alloys." He says the two projects seek an understanding of microstructure evolution in titanium alloys and resulting properties, and that the funding for both was obtained because of his interactions with the US Air Force Research Laboratory and AFOSR staff members.

"A former colleague of mine at the Max Planck Institute for Iron Research in Germany told me about this program," says Pyuck-Pa Choi, associate professor of materials science and engineering at the Korea Advanced Institute of Science and Technology. "The program seemed to be a very good opportunity for me to acquire funding for fundamental research on high-temperature materials."

Choi received a grant of $\$ 50,000$ per year for three years for research on engineering alloys for structural applications, in particular high-temperature applications.

"I thought that a proposal to develop new cobalt-based superalloys for aerospace gas turbine applications would fit very well to my research portfolio as well as to the research interests of the US Air Force and AOARD," Choi says.

AOARD grant sizes vary depending on the specific location, type of research (theoretical versus experimental), and project length. The median award size is $\$ 58,000$ for 12 months. One type of award is a one-year seed grant for proof-of-concept studies; other types of grants are given for scientific workshops and conferences.

And AOARD stays in touch with the researchers they fund.

"I have regular contact with my program managers and also occasionally with other program managers for AFOSR - they are very professional people and they are all scientists - some civilian and some military," Iacopi says. "They do not apply to AFOSR for funding of their own research, so their judgment is unbiased by competition."

"The contact was extensive during the phase of project submission and the experience was excellent. I found the program officers very supportive," Banerjee says.

Iacopi says she encourages colleagues seeking funding for fundamental research with potential for a good payoff to consider one of the international programs of AFOSR, including AOARD.

"AFOSR prides itself on having supported over 70 Nobel Prize [laureates], from all over the world, early in their careers - which is admirable as it is always riskier to support early career researchers," Iacopi says.

William G. Schulz

EC's Horizon 2020 funds development of organ-on-chip technology www.h2020-orchid.eu

$\mathrm{W}$

ith funding from the European Commission's Horizon 2020 program, a consortium led by Leiden University Medical Center and the Dutch Institute for Human Organ and Disease Model Technologies is creating a roadmap for the future development of organ-on-chip technology, aiming at establishing a European infrastructure to enable coordinated development, production, and implementation of organ-on-chip systems. Called ORCHID, the consortium will facilitate dialogue toward accelerating the development of prototypes of organs on chips (validated cell systems that mimic diseased or healthy human tissue), and the implementation of this technology by a broad group of potential users in science, health care, and industry. Organon-chip technology will deliver applications in personalized medicine and safety pharmacology, and offer alternatives to conventional animal testing.

ORCHID's aim is to build an infrastructure for scientists, policymakers, funders, and end users to join the decision-making processes that will direct future European developments in organon-chip applications. An essential contribution of ORCHID will be the establishment of a digital platform enabling knowledge sharing between researchers and representatives of private corporations, including insurance companies, pharmaceutical and biotech companies, food industry, health foundations, and patient organizations. This platform will provide overviews and updates of current and new organ-on-chip initiatives so that users can track progress, consult developers directly, and identify gaps in present knowledge, limiting implementation. ORCHID will also address ethical and regulatory issues, particularly concerning personalized information, economic and societal impact, training of researchers, and the design of a $R \& D$ roadmap. 